\title{
Frequency and syndrome specificity of antibodies to aquaporin-4 in neurological patients with rheumatic disorders
}

\author{
Sven Jarius', Christian Jacobi', Jerome de Seze ${ }^{2}$, \\ Helene Zephir ${ }^{3,4}$, Friedemann Paul ${ }^{5}$, Diego Franciotta ${ }^{6}$, \\ Paulus Rommer ${ }^{7}$, Simone Mader ${ }^{8}$, Ingo Kleiter', \\ Markus Reindl ${ }^{8}$, Gulsen Akman-Demir ${ }^{10}$, \\ Thomas Seifert-Held"', Wolfgang Kristoferitsch'2, \\ Arthur Melms ${ }^{13}$, Klaus-Peter Wandinger ${ }^{14,15}$ and \\ Brigitte Wildemann'
}

\begin{abstract}
Background: A new autoantibody (termed NMO-lgG, or AQP4-Ab) has recently been described in patients with neuromyelitis optica (NMO) and its formes frustes, longitudinally extensive transverse myelitis (LETM) and recurrent optic neuritis (rON). However, AQP4-Ab has been found also in patients with co-existing rheumatic diseases such as systemic lupus erythematosus (SLE) or Sjögren's syndrome (SS), conditions which are characterized by broad, polyspecific B cell activation.

Objectives: In this study, we aimed at evaluating the syndrome specificity and frequency of AQP4-Ab in patients with rheumatic diseases and neurological symptoms.

Methods: For this purpose, serum samples from 109 neurological patients with established connective tissue disorders (CTD) $(n=54)$, possible CTD $(n=42)$, or vasculitis $(n=13)$ were analysed for the presence of AQP4-Ab by a cellbased assay employing recombinant human AQP4.

Results: AQP4-Ab was detectable in $3 \mathrm{I} / 40$ (78\%) patients with CTD and NMO spectrum disorders (median titre, I:I000) but in none of the samples obtained from patients with CTD or vasculitis and neurological disorders other than NMO, LETM, or rON $(n=69)$.

Conclusion: The high syndrome specificity of the antibody for neuromyelitis optica spectrum disorders (NMOSDs) in patients with CTD supports the concept of AQP4-Ab being involved in the pathogenesis of these neurological conditions, and argues against AQP4-Ab simply being part of the polyclonal B cell activation generally associated with rheumatic diseases. Moreover, the finding that AQP4-Ab is present in patients with CTD and co-existing NMOSD with approximately the same frequency as in patients without CTD strengthens the case of CTD and AQP4-Ab positive NMOSD representing two co-existing yet distinct entities in the majority of patients.
\end{abstract}

\footnotetext{
'Division of Molecular Neuroimmunology, Department of Neurology, University of Heidelberg, Heidelberg, Germany.

${ }^{2}$ Clinique Neurologique, CHU de Strasbourg, Strasbourg, France.

${ }^{3}$ Pôle Neurologique, Hôpital Roger Salengro, CHRU de Lille, Lille, France.

${ }^{4}$ Laboratoire d'Immunologie, Université Lille Nord de France, Lille, France.

${ }^{5}$ NeuroCure Clinical Research Center, Charité - University Medicine Berlin, Berlin, Germany.

'IRCCS, National Neurological Institute 'C. Mondino', Pavia, Italy.

${ }^{7}$ Department of Neurology, Medical University of Vienna, Vienna, Austria.

${ }^{8}$ Clinical Department of Neurology, Innsbruck Medical University, Innsbruck, Austria.

${ }^{9}$ Department of Neurology, University Medical Centre Regensburg, Regensburg, Germany.

${ }^{10}$ Department of Neurology, University of Istanbul, Istanbul, Turkey.
}

\footnotetext{
"Department of Neurology, Graz Medical University, Graz, Austria.

${ }^{12}$ Department of Neurology, Sozialmedizinisches Zentrum - Donauspital, Vienna, Austria.

${ }^{13}$ Department of Neurology, University of Tuebingen, Tuebingen, Germany.

${ }^{14}$ Institute for Experimental Immunology, affiliated to Euroimmun, Luebeck, Germany.

${ }^{15}$ Institute for Neuroimmunology and Clinical MS Research, University Medical Center Eppendorf, Hamburg, Germany.

Corresponding author:

Brigitte Wildemann, MD, Division of Molecular Neuroimmunology, Department of Neurology, University of Heidelberg, Heidelberg, Germany

Email: Brigitte.Wildemann@med.uni-heidelberg.de
} 


\section{Keywords}

antibody to aquaporin-4, connective tissue disorders, diagnosis, longitudinally extensive transverse myelitis, neuromyelitis optica (Devic's disease), neuropsychiatric lupus, NMO-lgG, rheumatic diseases, scleroderma, Sjögren's syndrome, systemic lupus erythematosus, vasculitis

Date received: 7th July 2010; revised: 19th December 2010; 20th February 20II; accepted: 22nd February 20II

\section{Introduction}

Neuromyelitis optica (NMO) is a very rare inflammatory disorder of the central nervous system (CNS) of putative autoimmune aetiology, which mainly affects the optic nerves and spinal cord. ${ }^{1}$ Recent studies demonstrated that NMO and its formes frustes, longitudinally extensive transverse myelitis (LETM) ${ }^{2}$ and recurrent optic neuritis $(\mathrm{rON}),{ }^{3,4}$ are associated with the presence of a newly detected serum reactivity to structures adjacent to the CNS microvasculature and pia mater in $60-80 \%$ of cases (called NMO- $\mathrm{IgG}$ ). ${ }^{5,6}$ This antibody was later shown to target aquaporin-4 (AQP4), the most abundant water channel in the $\mathrm{CNS}^{7,8}$

Interestingly, AQP4-Ab was found also in a subset of NMO and LETM patients with co-existing connective tissue disorders (CTDs) such as systemic lupus erythematosus (SLE) or Sjögren's syndrome (SS). ${ }^{9}$ However, CTDs are usually associated with a broad repertoire of autoantibodies, many of which are of no proven pathogenic impact. AQP4-Ab could be part of that polyclonal B-cell response in patients with CTD rather than being pathogenetically related to the neurological syndrome.

Strong evidence for a role of AQP4-Ab in the pathogenesis of NMO and LETM/rON in patients with CTD would come from the demonstration that the antibody is present only in patients with CTD and NMO or LETM/rON but not in patients with CTD and neurological disorders other than NMO and LETM/rON.

Here we report on the AQP4-Ab serostatus of 109 patients with established or suspected rheumatic diseases and co-existing neurological disorders.

\section{Patients and methods}

Inclusion criterion was the presence of SLE, SS, systemic scleroderma, polymyositis/dermatomyositis, Sharp syndrome, or vasculitis at the time of neurological presentation, or of autoantibodies usually associated with those disorders. Exclusion criterion was the presence of co-existing conditions that could sufficiently explain the neurological syndrome, such as infections, or stroke due to well established causes other than CTD. Group I consisted of 54 patients with definite CTD and neurological involvement (see Table 1 for details). CTD in this group comprised SLE $(n=41)$, primary SS (6), SLE with secondary SS (2), systemic sclerosis (1), systemic sclerosis with SS (1), scleroderma en coup-de-sabre (1), CREST syndrome (1), and Sharp syndrome with biopsy proven polymyositis (1). Group II consisted of 42 patients with various neurological syndromes (Table 1), who were positive for auto-antibodies usually associated with CTD but who did not fulfil the formal criteria for any CTD based on the data available for analysis. Anti-nuclear antibodies were present in 40/42, SS-A and/or SS-B in 13, cardiolipin and anti-B2-glycoprotein antibodies in 16, double stranded DNA antibodies in nine, ribonucleoprotein antibodies in nine, lupus anticoagulans in three, Scl-70 antibodies in two, histon antibodies in three, and centromer antibodies in five; three patients were, in addition, positive for rheumatoid factor and one for single strand DNA antibodies and circulating immune complexes. Further features of CTD such as Raynaud's phenomenon, sicca symptoms, polyserositis, nephritis, or arthralgia were present in 16 patients; in addition, Coombs' positive anaemia was reported in one, and other haematological disturbances in seven. Group III consisted of 13 patients with vasculitis. Seven patients had primary arteritis of the CNS (PACNS), four giant cell arteritis, one leukocytoclastic vasculitis, and one post-infectious systemic vasculitis involving the CNS.

All SLE patients fulfilled the American College of Rheumatology (ACR) criteria. ${ }^{10,11}$ SS was diagnosed according to Vitali et al. ${ }^{12}$ and scleroderma according to Masi et al. ${ }^{13}$ The diagnosis of PACNS was established according to Moore and Richardson ${ }^{14}$ in 5/7 cases and according to Calabrese and Mallek ${ }^{15}$ in two cases. NMO was diagnosed according to Wingerchuk's 2006 criteria. ${ }^{1}$ LETM was defined as myelitis extending over three or more vertebral segments. NMOSD followed a relapsing course in 36 patients and was monophasic in four.

Samples were tested for AQP4-Ab in an anonymized fashion at the Department of Neurology, University of Heidelberg by means an indirect immunofluorescence assay employing human full length AQP4 expressed 
Table I. Rheumatic diagnoses and neurological syndromes, epidemiological data and serum AQP4-Ab results from neurological patients with established or possible connective tissue disorders or vasculitis

\begin{tabular}{lllll}
\hline Diagnostic groups and neurolog- & & & & \\
ical syndromes & $N$ & Sex (m:f) & Age (median, range) & AQP4-AbN (\%) \\
\hline Definite CTD & 54 & $1: 20$ & $46(16-75)$ & $16(30)$ \\
NMO spectrum disorders & 21 & & & $16(76)$ \\
NMO & 15 & & & $10(67)$ \\
LETM & 5 & & $5(100)$ \\
rON & 1 & & $1(100)$ \\
Syndromes other than NMO/ & 33 & & & $0(0)$ \\
LETM/rON & 42 & $1: 3.6$ & $45(21-79)$ & $15(36)$ \\
Possible CTD & 19 & & & $15(79)$ \\
NMO spectrum disorders & 12 & & & $10(83)$ \\
NMO & 6 & & $4(67)$ \\
LETM & 1 & & $1(100)$ \\
rON & 23 & & & $0(0)$ \\
Syndromes other than NMO/ & & $52(35-88)$ & $0(0)$ \\
LETM/rON & 13 & $1: 12$ & & \\
Vasculitis & & & \\
\hline
\end{tabular}

NMO: neuromyelitis optica, LETM: longitudinally extensive transverse myelitis, rON: recurrent optic neuritis.

in HEK293 cells. ${ }^{16}$ Briefly, formalin-fixed AQP4-transfected HEK 293 cells, which were provided immobilized on microscopy slides, were incubated with serum or CSF diluted $1: 10$ in $1 \%$ bovine serum albumin and, after washing in PBS, with a goat anti-human IgG antibody conjugated to fluorescein isothiocyanate (FITC). After final washing in PBS and mounting in glycerol containing an anti-fading agent, cells were analysed for bound AQP4-IgG on a Nikon 90i fluorescence microscope (Nikon Imaging Center, University of Heidelberg, Germany). This assay was previously shown to have a sensitivity of $78 \%$ and a specificity of $100 \%(n=151){ }^{16}$ All patients were of Caucasian origin. Sex ratios in the various diagnostic groups as well as median age at blood sampling are given in Table 1 .

\section{Results}

AQP4-Ab was detectable in 20/27 (74\%) samples from patients with definite or possible rheumatic diseases and $\mathrm{NMO}$, in $11 / 13(85 \%)$ samples from patients with rheumatic diseases and LETM or rON, but in none out of 69 samples obtained from patients with rheumatic diseases and neurological disorders other than NMO, LETM, or rON $(p<0.00001$; Fisher exact test). Neurological disorders or symptoms in the latter group included longitudinally non-extensive transverse myelitis, demyelinating disease of the brain, brain infarction, (micro)bleedings of the brain, aseptic meningitis, seizures, trigeminal neuralgia, polyneuropathy, mononeuritis, ataxia, depression and other psychiatric syndromes, neuropsychological disorders, myositis, and headache.

The frequency of AQP4-Ab was $84.6 \%$ in patients with SLE and NMO or LETM/rON and $62.5 \%$ in patients with other CTD and NMO or LETM. AQP4-Ab serum titres in patients with NMOSD and CTD (median, 1:1000; range, 1:50-1:10.000; $n=19$; definite CTD in 16, possible CTD in three) did not differ significantly from those in a group of unselected control patients with NMOSD but no CTD (median, $1: 1000$; range, $1: 50-1: 12.500 ; n=20)$. AQP4-Ab was positive in $78 \%(28 / 36)$ of the relapsing cases, and in $75 \%(3 / 4)$ of the monophasic cases. The median maximum longitudinal extension of spinal cord lesions did not differ between AQP4-Ab positive and AQP4-Ab negative NMOSD patients (5 [range, 3-19] and 5.5 [3 to 'entire spinal cord'], respectively), nor did the median total number of relapses (4.5 and 6 , respectively).

Cranial lesions as detected by MRI were present in $17 / 40(43 \%)$ NMOSD patients, with no significant difference between AQP4-Ab positive (12/31) and negative cases (5/9). Brain stem MRI lesions were noted in 6/31 AQP4-Ab positive NMOSD patients (19\%) and in $3 / 9$ AQP4-Ab negative NMOSD patients (33\%), and were situated in the medulla oblongata in six patients (AQP4-Ab positive in five) and in the pons in two (AQP4-Ab positive in one). Brain stem involvement was also suspected in some additional AQP4-Ab positive patients based on clinical presentation (vomiting and tonic fits in one, and diplopia in two), though 
MRI was negative. In two patients with brain stem lesions, both of which were AQP4-Ab positive, additional lesions in the diencephalon (thalamus in two, hypothalamus in one), mesencephalon (cerebral peduncle), basal ganglia (capsula interna), and cerebellar peduncles were observed; in addition, cerebellar lesions were evident in one of them. Supratentorial white matter MRI lesions were noted in $32 \%(10 / 31)$ of the AQP4-Ab positive and 22\% (2/9) of the AQP4-Ab negative NMOSD patients. Periventricular, juxtacortical, or callosal lesions were reported in six of the patients (AQP4-Ab positive in five). Extra-opticospinal neurological manifestations, which are normally absent in patients with $\mathrm{NMO},{ }^{20}$ were documented in six AQP4$\mathrm{Ab}$ positive patients and one AQP4-Ab negative patient. These manifestations included psychosis in two (though steroid associated in one of them), cognitive deficits in one, seizures in one, transient ischemic attacks in two, polyneuropathy in two, and polymyositis in one. Three NMOSD patients (AQP4-Ab positive in two) had co-existing acetylcholine receptor antibody positive myasthenia gravis, one of which in addition suffered from coeliac disease.

Thryoid stimulating hormone receptor antibody positive autoimmune thyroiditis with hyperthyroidism (Grave's disease) was present in four patients (AQP4$\mathrm{Ab}$ positive in three) and Hashimoto thyroiditis with hypothyroidism in two (both AQP4-Ab positive). In two additional patients, thyroglobulin or thyroid peroxidase antibodies respectively, but no evidence for thyroid dysfunction, were found, and in two further patients hypothyrodism was noted at least once but anti-thyroid antibodies had not been determined (all AQP4-Ab positive).

One AQP4-Ab positive patient with NMOSD in group I and three AQP4-Ab positive patients with NMOSD in group II were positive for perinuclear antineutrophil cytoplasmic antibodies (pANCA) (myeloperoxidase-specific pANCA in two, pANCA of unknown specificity in two); in contrast, 26 other patients with NMOSD (AQP4-Ab positive in 20) were negative for ANCAs. A strong female preponderance was evident both in the NMOSD (sex ratio, m:f = $1: 12.3)$ and in the non-NMOSD ( $\mathrm{m}: \mathrm{f}=1: 4.6)$ group $(p=0.23)$, with no significant difference between AQP4-Ab positive (female in 97\%) and AQP4-Ab negative NMOSD patients (female in $78 \%$ ). See Table 1 for further results.

\section{Discussion}

We found that AQP4-Ab seropositivity in patients with CTDs such as SLE or SS and co-existing neurological disorders is restricted to those with NMO, LETM, or $\mathrm{rON}$. The high syndrome specificity of AQP4-Ab in patients with neuropsychiatric CTD argues strongly against the antibody being simply part of the CTDassociated spectrum of non-tissue specific autoantibodies but suggests that it is linked to the pathogenesis of NMO, LETM, or rON in those patients. In previous studies, we and others had already demonstrated a lack of AQP4-Ab seropositivity in patients with CTD but no neurological disease $(n=45){ }^{8,9,17}$ We also found that both the rate of AQP4-Ab seropositivity and the median AQP4-Ab serum titres do not differ significantly between patients with NMOSD and co-existing CTD and patients with NMO but no CTD.

Our results corroborate findings from two smaller studies. Pittock et al. reported a lack of AQP4-Ab in eight patients with CTD and neurological syndromes other than NMOSD from an American cohort and in six patients from a French cohort. ${ }^{9}$ In this study, a nonAQP4-specific immunohistochemical assay employing adult mouse cerebellum tissue sections as substrate was used to detect AQP4-Ab. In a second, independent study, Wandinger et al. very recently reported a lack of AQP4-Ab in a mixed British-German cohort with neurological syndromes but not NMOSD. ${ }^{17}$ This study, which employed the same recombinant assay as used here, included 41 patients with CTD and neurological syndromes other than NMOSD, but only three patients with CTD and NMO (compared with 27 in the present study).

The finding of AQP4-Ab in a subset of patients with CTD and NMO, LETM, or rON identifies these cases as part of the newly described spectrum of autoimmune AQP4-channelopathies. ${ }^{7,18,19}$ Future trials on neurological involvement in CTD have to take into account that AQP4-Ab positive patients may represent a distinct subgroup with differential pathogenesis and treatment response. Similarly, this finding has implications for everyday clinical practice. AQP4-Ab positive LETM or rON usually takes a relapsing course and confers a high risk of conversion to NMO, which, if untreated, often results in irreversible blindness and immobility within a short time. ${ }^{2,4,20}$ Although no controlled treatment trials exist in NMO due to the rarity of the disorder, long term immunosuppression, e.g. with azathioprine or rituximab, is thought to be crucial in AQP4-Ab positive patients, and plasma exchange seems to be beneficial for the treatment of acute relapses. $^{21-25}$

The reason why AQP4-Ab positive NMO/LETM is frequently associated with CTD is unclear. It has been speculated that the co-existence of the two disorders in the same patient might reflect a general autoimmune predisposition. The concept of coexisting independent, antibody-mediated autoimmune disorders in the same patient is further supported by the association of AQP4$\mathrm{Ab}$ positive NMOSD with autoimmune conditions such 
as Grave's disease, myasthenia gravis, or coeliac disease, as observed in this study as well as in previous ones. ${ }^{26-30}$

In addition, CTD-induced tissue damage might well promote AQP4-Ab-induced pathology. There is increasing evidence for a direct impact of AQP4-Ab in the immunopathogenesis of NMO/LETM. ${ }^{18,19,31-33}$ Briefly, the antibody is thought to confer tissue damage by complement activation and induction of a cellular immune response. ${ }^{31,32,34-38}$ However, several findings indicate that AQP4-Ab may not be sufficient to cause CNS disease on its own. First, AQP4-Ab remains detectable in patients with NMO during remission, partly at high titres, ${ }^{39}$ and is even detectable many years prior to disease onset in some patients. ${ }^{40,41}$ Secondly, in animal models of NMO, passive transfer of the antibody into rodents had no effect unless disruption of the blood-brain barrier or brain inflammation was induced. ${ }^{31,32,42}$

Importantly, aquaporin-4, the target antigen of AQP4-Ab, is an integral constituent of the blood-brain barrier. The protein is concentrated in the astrocytic endfeet sealing the brain parenchyma against the brain vasculature. Vascular damage induced by CTD-associated vasculitis might thus both render AQP4 accessible to AQP4-Ab and, in addition, create the inflammatory environment required for induction of the AQP4Ab-mediated inflammatory cascade. Interestingly, four AQP4-Ab positive patients with NMO/LETM were positive for pANCA, antibodies frequently associated with vasculitis, in the present study.

It should be underlined, however, that CTD, though a possible promoter, is not a prerequisite of disease activity in NMO. Pittock et al. ${ }^{9}$ systematically investigated the frequency of ANA as a sensitive and early marker of CTD associated autoimmunity, and could demonstrate a lack of ANA positivity in around $40 \%$ of AQP4-Ab positive NMOSD patients. Accordingly, the onset of NMOSD preceded the occurrence of clinical or serological signs of CTD related autoimmunity in at least five of our patients, who only later in the disease course developed SLE ( $n=3$; AQP4-Ab positive in two) or SS ( $n=2$; AQP4-Ab positive in one). Moreover, the priority of CTD in time noted in many patients does not necessarily imply a causal relationship. Instead, at least in patients with SLE, it might simply reflect the differential peaks of onset of the two conditions (30 years of age in $\mathrm{SLE},{ }^{43} 40$ in relapsing $\mathrm{NMO}^{20}$ ).

Importantly, 9/39 patients with NMOSD were negative for AQP4-Ab in our study, suggesting that NMOSD might be aetiologically heterogeneous. In a previous study, we found AQP4-Ab in 95/96 serum samples that were obtained during remission and under treatment with immunosuppressants such as azathioprine, rituximab, cyclophosphamide, mitoxantrone, or dexamethasone, rendering it unlikely that AQP4-Ab seronegativity in those nine patients was caused by treatment effects. ${ }^{39}$ Alternatively, tissue damage might be caused by CTD-mediated mechanisms (e.g. vasculitis) in those patients. Moreover, so far unknown autoantibodies might play a role, as indicated by the fact that plasma exchange was found to be beneficial also in some AQP4-Ab negative patients in a recent study. ${ }^{44}$

Seven patients had extra-opticospinal disorders such as PNP or seizures, which are not usually associated with AQP4-autoimmunity. The presence of extraopticospinal signs and symptoms should prompt physicians to check for co-existing CTD.

Given the number of centres involved in this study, we cannot fully exclude that some sort of sampling bias might have influenced the data on AQP4-Ab frequency in CTD and NMO spectrum disorders. However, this study did not primarily aim at assessing the frequency of AQP4-Ab but the syndrome specificity of this new antibody in patients with CTD. Moreover, the frequency found in this study $(77 \%)$ is in good accordance with data from two smaller previous studies. ${ }^{9,17}$

It is of note that antibodies to AQP4 in patients with CTD and NMO/LETM were investigated by means of a recombinant assay instead of immunohistochemistry (IHC) in this study. Recombinant assays have repeatedly been demonstrated to be more sensitive and slightly more specific than IHC. ${ }^{16,35,45-48}$ The test used in this study had a $12.5 \%$ higher sensitivity when compared with standard IHC on adult mouse cerebellum sections and a specificity of $100 \%(n=151) .{ }^{16}$

In conclusion, our data demonstrates that AQP4-Ab is highly specific for NMO/LETM in patients with $\mathrm{CTD}$. This finding strengthens the case of AQP4-Ab being involved in the pathogenesis of NMO/LETM in patients with CTD. AQP4-Ab testing should be performed in all patients with CTD presenting with signs and symptoms suggestive of NMO, LETM, or ON.

\section{Acknowledgements}

The cells used in this study were kindly provided by Euroimmun, Luebeck, Germany. We are grateful to Professor Maria K Storch (Medical University of Graz) and Dr Franziska Di Pauli (Medical University of Innsbruck) for their support and cooperation, and to Mrs Anna Eschlbeck, Mrs Brigitte Fritz, and the Nikon Imaging Center at the University of Heidelberg for excellent technical assistance.

\section{Funding}

This work was supported by a Research Fellowship from the European Committee for Treatment and Research in Multiple Sclerosis (ECTRIMS) to SJ, research grants from Bayer Schering Pharma and from Merck Serono to BW, a grant from the German Research Foundation (DFG; Cluster of Excellence 257) to FP, and a research grant of the Austrian multiple sclerosis research society to MR. 


\section{Conflict of interest statement}

KPW is an employee of Euroimmun, Luebeck, Germany. Euroimmun provided the cells used in this study, but had no influence on study design, analysis or interpretation of data, or the decision to publish this manuscript. The other authors declare no competing financial interest.

\section{References}

1. Wingerchuk DM, Lennon VA, Pittock SJ, Lucchinetti $\mathrm{CF}$ and Weinshenker BG. Revised diagnostic criteria for neuromyelitis optica. Neurology 2006; 66: 1485-1489.

2. Weinshenker BG, Wingerchuk DM, Vukusic S, et al. Neuromyelitis optica IgG predicts relapse after longitudinally extensive transverse myelitis. Ann Neurol 2006; 59: 566-569.

3. Matiello M, Lennon VA, Jacob A, et al. NMO-IgG predicts the outcome of recurrent optic neuritis. Neurology 2008; 70: 2197-2200.

4. Jarius S, Frederikson J, Waters P, Paul F, Akman-Demir G, Marignier R, et al. Frequency and prognostic impact of antibodies to aquaporin-4 in patients with optic neuritis. J Neurol Sci 2010; 298(1-2): 158-162.

5. Lennon VA, Wingerchuk DM, Kryzer TJ, et al. A serum autoantibody marker of neuromyelitis optica: distinction from multiple sclerosis. Lancet 2004; 364: 2106-2112.

6. Jarius S, Franciotta D, Bergamaschi R, et al. NMO-IgG in the diagnosis of neuromyelitis optica. Neurology 2007; 68: 1076-1077.

7. Lennon VA, Kryzer TJ, Pittock SJ, Verkman AS and Hinson SR. IgG marker of optic-spinal multiple sclerosis binds to the aquaporin-4 water channel. J Exp Med 2005; 202: 473-477.

8. Paul F, Jarius S, Aktas O, et al. Antibody to aquaporin 4 in the diagnosis of neuromyelitis optica. PLoS Med 2007; 4: e133.

9. Pittock SJ, Lennon VA, de Seze J, et al. Neuromyelitis optica and non organ-specific autoimmunity. Arch Neurol 2008; 65: 78-83.

10. Hochberg MC. Updating the American College of Rheumatology revised criteria for the classification of systemic lupus erythematosus. Arthritis Rheum 1997; 40: 1725.

11. Tan EM, Cohen AS, Fries JF, et al. The 1982 revised criteria for the classification of systemic lupus erythematosus. Arthritis Rheum 1982; 25: 1271-1277.

12. Vitali C, Bombardieri S, Jonsson R, et al. Classification criteria for Sjogren's syndrome: a revised version of the European criteria proposed by the American-European Consensus Group. Ann Rheum Dis 2002; 61: 554-558.

13. Masi AT, Rodnan GP, Medsger TA, Altman RD, et al. Preliminary criteria for the classification of systemic sclerosis (scleroderma). Subcommittee for scleroderma criteria of the American Rheumatism Association Diagnostic and Therapeutic Criteria Committee. Arthritis Rheum 1980; 23: 581-590.

14. Moore PM and Richardson B. Neurology of the vasculitides and connective tissue diseases. J Neurol Neurosurg Psychiatry 1998; 65: 10-22.
15. Calabrese LH and Mallek JA. Primary angiitis of the central nervous system. Report of 8 new cases, review of the literature, and proposal for diagnostic criteria. Medicine (Baltimore) 1988; 67: 20-39.

16. Jarius S, Probst C, Borowski K, et al. Standardized method for the detection of antibodies to aquaporin-4 based on a highly sensitive immunofluorescence assay employing recombinant target antigen. J Neurol Sci 2010; 291: 52-56.

17. Wandinger KP, Stangel $M$, Witte $T$, et al. Autoantibodies against aquaporin-4 in patients with neuropsychiatric systemic lupus erythematosus and primary Sjogren's syndrome. Arthritis Rheum 2010; 62: 1198-1200.

18. Jarius S, Paul F, Franciotta D, et al. Mechanisms of disease: aquaporin-4 antibodies in neuromyelitis optica. Nat Clin Pract Neurol 2008; 4: 202-214.

19. Jarius S and Wildemann B. AQP4 antibodies in neuromyelitis optica: pathogenic and diagnostic relevance. Nature Rev Neurol 2010; 6: 383-392.

20. Wingerchuk DM, Hogancamp WF, O'Brien PC and Weinshenker BG. The clinical course of neuromyelitis optica (Devic's syndrome). Neurology 1999; 53: 1107-1114.

21. Cree BA, Lamb S, Morgan K, Chen A, Waubant E and Genain C. An open label study of the effects of rituximab in neuromyelitis optica. Neurology 2005; 64: 1270-1272.

22. Jacob A, Weinshenker BG, Violich I, et al. Treatment of neuromyelitis optica with rituximab: retrospective analysis of 25 patients. Arch Neurol 2008; 65: 1443-1448.

23. Mandler RN, Ahmed W and Dencoff JE. Devic's neuromyelitis optica: a prospective study of seven patients treated with prednisone and azathioprine. Neurology 1998; 51: 1219-1220.

24. Keegan M, Pineda AA, McClelland RL, Darby $\mathrm{CH}$, Rodriguez $\mathrm{M}$ and Weinshenker BG. Plasma exchange for severe attacks of CNS demyelination: predictors of response. Neurology 2002; 58: 143-146.

25. Nakashima I, Takahashi $T$, Nishiyama $S$, et al. Plasma exchange for neuromyelitis optica with aquaporin-4 antibody. Neurology 2009; 72: A187.

26. Kay CS, Scola RH, Lorenzoni PJ, Jarius S, Arruda WO and Werneck LC. NMO-IgG positive neuromyelitis optica in a patient with myasthenia gravis but no thymectomy. J Neurol Sci 2008; 275: 148-150.

27. McKeon A, Lennon VA, Jacob A, et al. Coexistence of myasthenia gravis and serological markers of neurological autoimmunity in neuromyelitis optica. Muscle Nerve 2009; 39: 87-90.

28. Bergamaschi R, Jarius S, Robotti M, Pichiecchio A, Wildemann B and Meola G. Two cases of benign neuromyelitis optica in patients with celiac disease. J Neurol 2009.

29. Jacob S, Zarei M, Kenton A and Allroggen H. Gluten sensitivity and neuromyelitis optica: two case reports. J Neurol Neurosurg Psychiatry 2005; 76: 1028-1030.

30. Jarius S, Jacob S, Waters P, Jacob A, Littleton E and Vincent A. Neuromyelitis optica in patients with gluten 
sensitivity associated with antibodies to aquaporin-4. J Neurol Neurosurg Psychiatry 2008; 79: 1084.

31. Bradl M, Misu T, Takahashi T, et al. Neuromyelitis optica: pathogenicity of patient immunoglobulin in vivo. Ann Neurol 2009; 66: 630-643.

32. Bennett JL, Lam C, Kalluri SR, et al. Intrathecal pathogenic anti-aquaporin-4 antibodies in early neuromyelitis optica. Ann Neurol 2009; 66: 617-629.

33. Saadoun S, Waters P, Bell BA, Vincent A, Verkman AS and Papadopoulos MC. Intra-cerebral injection of neuromyelitis optica immunoglobulin $\mathrm{G}$ and human complement produces neuromyelitis optica lesions in mice. Brain 2010; 133: 349-361.

34. Hinson SR, Pittock SJ, Lucchinetti CF, et al. Pathogenic potential of $\mathrm{IgG}$ binding to water channel extracellular domain in neuromyelitis optica. Neurology 2007; 69: 2221-2231.

35. Waters P, Jarius S, Littleton E, et al. Aquaporin-4 antibodies in neuromyelitis optica and longitudinally extensive transverse myelitis. Archives of neurology 2008; 65: 913-919.

36. Vincent T, Saikali P, Cayrol R, et al. Functional consequences of neuromyelitis optica-IgG astrocyte interactions on blood-brain barrier permeability and granulocyte recruitment. J Immunol 2008; 181: 5730-5737.

37. Saadoun S, Waters P, Bell BA, Vincent A, Verkman AS and Papadopoulos MC. Intra-cerebral injection of neuromyelitis optica immunoglobulin $\mathrm{G}$ and human complement produces neuromyelitis optica lesions in mice. Brain 2010; 133: 349-361.

38. Kinoshita M, Nakatsuji Y, Kimura T, et al. Anti-aquaporin-4 antibody induces astrocytic cytotoxicity in the absence of CNS antigen-specific T cells. Biochem Biophys Res Commun 2010.

39. Jarius S, Aboul-Enein F, Waters P, et al. Antibody to aquaporin-4 in the long-term course of neuromyelitis optica. Brain 2008; 131: 3072-3080.
40. Nishiyama S, Ito T, Misu T, et al. A case of NMO seropositive for aquaporin-4 antibody more than 10 years before onset. Neurology 2009; 72: 1960-1961.

41. Jarius S, Paul F, Martins da Silva A, et al. Neuromyelitis optica and longitudinally extensive transverse myelitis following thymectomy for myasthenia gravis. Mult Scler 2007; 13: P534.

42. Kinoshita M, Nakatsuji Y, Kimura $T$, et al. Neuromyelitis optica: passive transfer to rats by human immunoglobulin. Biochem Biophys Res Commun 2009; 386: 623-627.

43. Cervera R, Khamashta MA and Hughes GR. The Eurolupus project: epidemiology of systemic lupus erythematosus in Europe. Lupus 2009; 18: 869-874.

44. Bonnan M, Valentino R, Olindo S, Mehdaoui H, Smadja $\mathrm{D}$ and Cabre P. Plasma exchange in severe spinal attacks associated with neuromyelitis optica spectrum disorder. Mult Scler 2009; 15: 487-492.

45. Takahashi T, Fujihara K, Nakashima I, et al. Anti-aquaporin-4 antibody is involved in the pathogenesis of NMO: a study on antibody titre. Brain 2007; 130 : 1235-1243.

46. Matsushita T, Isobe N, Matsuoka T, et al. Aquaporin-4 autoimmune syndrome and anti-aquaporin-4 antibodynegative opticospinal multiple sclerosis in Japanese. Mult Scler 2009; 15: 834-847.

47. Chan KH, Kwan JS, Ho PW, Ho JW, Chu AC and Ramsden DB. Aquaporin-4 autoantibodies in neuromyelitis optica spectrum disorders: comparison between tissue-based and cell-based indirect immunofluorescence assays. J Neuroinflammation 2010; 7: 50 .

48. Mader S, Lutterotti A, Di Pauli F, et al. Patterns of antibody binding to aquaporin-4 isoforms in neuromyelitis optica. PLoS One 2010; 5: e10455. 